\title{
CRISPR/Cas9-edited HPV16/18 E6/E7 Plasmid
}

National Cancer Institute

\section{Source}

National Cancer Institute. CRISPR/Cas9-edited HPV16/18 E6/E7 Plasmid. NCI Thesaurus. Code C148185.

A plasmid encoding for clustered regularly interspaced short palindromic repeats (CRISPR) targeting the promoters for the human papillomavirus (HPV) type 16 (HPV16) and 18 (HPV18) epitopes E6 and E7, and coupled to the endonuclease cas9, with potential antineoplastic activities. Upon administration and transfection of the CRISPR/Cas9-edited HPV16/18 E6/E7 plasmid, the guide RNA (gRNA) of the CRISPR moiety specifically targets and binds to complementary sites on the HPV 16/18 promoter regions for E6 and E7. Cas9 cleaves these specific DNA sites, thereby disrupting HPV16/18 E6/E7 transcription. Decreased expression of E6 and E7 induces apoptosis and decreases tumor cell proliferation in HPV-driven tumor cells. This induces the expression of certain tumor suppressor genes, such as p53 and retinoblastoma 1 (RB1), which induces tumor cell apoptosis and inhibits tumor cell proliferation. 